\title{
Reynolds number and wind tunnel wall effects on the flow field around a generic UHBR engine high-lift configuration
}

\author{
Junaid Ullah ${ }^{1} \cdot$ Aleš Prachař ${ }^{2} \cdot$ Miroslav Šmíd ${ }^{2} \cdot$ Avraham Seifert $^{3} \cdot$ Vitaly Soudakov $^{4} \cdot$ Thorsten Lutz $^{1} \cdot$ Ewald Krämer $^{1}$
}

Received: 9 January 2020 / Revised: 2 July 2020 / Accepted: 18 August 2020 / Published online: 31 August 2020

(c) The Author(s) 2020

\begin{abstract}
RANS simulations of a generic ultra-high bypass ratio engine high-lift configuration were conducted in three different environments. The purpose of this study is to assess small scale tests in an atmospheric closed test section wind tunnel regarding transferability to large scale tests in an open-jet wind tunnel. Special emphasis was placed on the flow field in the separation prone region downstream from the extended slat cut-out. Validation with wind tunnel test data shows an adequate agreement with CFD results. The cross-comparison of the three sets of simulations allowed to identify the effects of the Reynolds number and the wind tunnel walls on the flow field separately. The simulations reveal significant blockage effects and corner flow separation induced by the test section walls. By comparison, the Reynolds number effects are negligible. A decrease of the incidence angle for the small scale model allows to successfully reproduce the flow field of the large scale model despite severe wind tunnel wall effects.
\end{abstract}

Keywords UHBR engine $\cdot$ Flow separation $\cdot$ Reynolds number effects $\cdot$ Wind tunnel wall effects

\section{Introduction}

The annual increase of around $4.8 \%$ in air passenger transport and the related impact on greenhouse gas emission and local air quality poses substantial challenges for the aircraft industry. Aviation already accounts for $2 \%$ of global greenhouse gas emission. If aviation would be considered as a country it would rank among the top ten emitting countries of the world [1]. In order to reduce the environmental footprint of aviation the European Union (EU) has set the ambitious goal in its report "Flightpath 2050" to cut $\mathrm{CO}_{2}$ and $\mathrm{NO}_{x}$ emissions by 75 and $95 \%$, respectively, until 2050 compared to those of the year 2000 [2]. Ultra-high bypass ratio (UHBR) engines

Junaid Ullah

ullah@iag.uni-stuttgart.de

1 Faculty 6: Aerospace Engineering and Geodesy (F06), Institute of Aerodynamics and Gas Dynamics, University of Stuttgart, Pfaffenwaldring 21, 70569 Stuttgart, Germany

2 VZLU-Czech Aerospace Research Centre, Beranovych 130, 19905 Prague, Czech Republic

3 School of Mechanical Engineering, Tel Aviv University, 69978 Tel Aviv, Israel

4 Central Aerohydrodynamic Institute (TsAGI), 140180 Zhukovsky, Moscow Region, Russia represent one opportunity to get closer to this goal, by maximizing the air mass flow passing through the bypass duct and thus reducing the thrust specific fuel consumption. The large nacelle diameter of such engines presents great challenges for aircraft designers, as compliance with ground clearance regulations require a close wing-nacelle coupling. To avoid collision with the nacelle during take-off and approach a large slat cut-out is inevitable. This geometric modification leads to premature flow separation on the wing and thus to an appreciable degradation of maximum lift. Established passive flow control measures like nacelle strakes [3] are not sufficient to compensate the losses associated with the missing slat section in the vicinity of the UHBR engine pylons [4]. Active flow control (AFC) has already demonstrated its ability regarding postponement of flow separation and is therefore a potential technology, which can be used to counteract the drawbacks caused by the extended slat cut-out. However, most of the AFC related studies focus on basic research, involving simplified models at low Reynolds number doubting their transferability to commercial aircraft. Additionally, conventional AFC actuators require a not feasible amount of energy or mass flow input. As a result, only a small number of AFC actuators reached a technology readiness level (TRL) high enough to be integrated in commercial aircraft. Therefore, within the scope of the EU Clean Sky2 project INAFLOWT [5], the goal was set to test 
the promising Suction and Oscillatory Blowing (SaOB) [6] actuator regarding its efficiency on a complex UHBR engine high-lift configuration at realistic inflow conditions. This demonstration on a near full-scale configuration will allow to increase the maturity level of technology toward TRL3, which means "verification of technology in a realistic environment". Beside the large scale wind tunnel tests, the scope of the project includes parametric AFC investigations on a downscaled small scale model (SSM). These tests will serve as a preliminary step to derive optimum AFC parameters for the final cost-intensive tests on the large scale model (LSM). The experimental results of the LSM as used in this study were provided by project partners from the Central Aerohydrodynamic Institute (TsAGI) based in Zhukovsky, Russia. Those tests were performed within in the framework of the AFloNext project [7]. Tel-Aviv University (Faculty of Engineering) was in charge of the recently conducted small scale tests. Since both wind tunnels are operated at atmospheric conditions the difference in the model scale is directly reflected in the Reynolds numbers. Additionally, the two wind tunnels differ regarding their test section enclosure-open (LSM) versus closed (SSM) test section environment.

In the present paper the Reynolds number effects and wind tunnel wall effects associated with the two different test conditions are studied independently by means of Computation Fluid Dynamics (CFD). For this purpose, three sets of simulations were conducted to allow an independent assessment of the two latter mentioned effects. First the LSM and the SSM were simulated at real prevailing environment and inflow conditions. The third set of simulations includes the LSM at modified Reynolds number (MR-LSM) corresponding to the small scale wind tunnel inflow conditions. The specific objective of this study is to qualify the SSM for representative tests with AFC by reproducing the baseline flow of the LSM. Thus the present work aims to pave the way for future extended experimental investigations of AFC in more affordable environments of non-cryogenic small scale wind tunnels. Section 2 of this paper contains a description of the research model and the wind tunnel campaigns. The subsequent section describes the numerical setup including the computational grids. Section 4 includes the computational results. In a first step, extended validations against wind tunnel data acquired from both test campaigns are conducted to encourage the subsequent derivation of flow characteristics from simulation results.

\section{Experimental setup}

\subsection{Research model}

The wind tunnel model used for the experiments and simulations was designed and manufactured for large-scale wind tunnel tests during the AFloNext project. This model was derived from a quasi-2D two-element high-lift configuration with deployed flap used in previous test campaigns within the European project SADE [8]. The model modifications comprised the introduction of a sweep angle of $28^{\circ}$ and an adjustment of the leading edge for the integration of a slat with extended cut-out in the center region. The final model incorporates the DLR F15-3eRef three-element airfoil [9] with flap and slat deployed at $28^{\circ}$ and $35^{\circ}$, respectively. The wind tunnel model represents a landing configuration. An UHBR engine was modeled by an underwing flow-through nacelle with a diameter of approx. $1.8 \mathrm{~m}$ mounted to the wing by a pylon. The inboard side of the nacelle is equipped with a strake. The wing has a reference chord length of about $3.26 \mathrm{~m}$ and a spanwise extension of about $5 \mathrm{~m}$ and hence corresponds to a 5:7.4 scale of the reference aircraft [10]. A 3D view of the model and the mounting arrangement is shown in Fig. 1. Deviations to the real approach flight of the reference aircraft comprise a difference in model scale and thus Reynolds number, differences arising form geometric simplifications, such as non-tapered wing, and differences in Mach number, due to maximum achievable velocities during the wind tunnel tests. However, numerical investigations within the AFLoNext project demonstrated that despite differences in stall behavior the location of flow separation and the effect of AFC regarding suppression of the latter is transferable between the real aircraft and the research model [10], which is crucial for the current project. Endplates were installed on the inboard and the outboard end of the wing to increase the effective wingspan. A leading-edge extension was added to the outboard slat leading edge corner to prevent premature corner separation on the outboard wing. The presence of corner flow separation would result in a deviant stall behavior and thus to a distortion of the measured force coefficients. This, in turn, could affect the assessment of AFC effects. A more in-depth description of the wind tunnel model can be found in $[11,12]$.

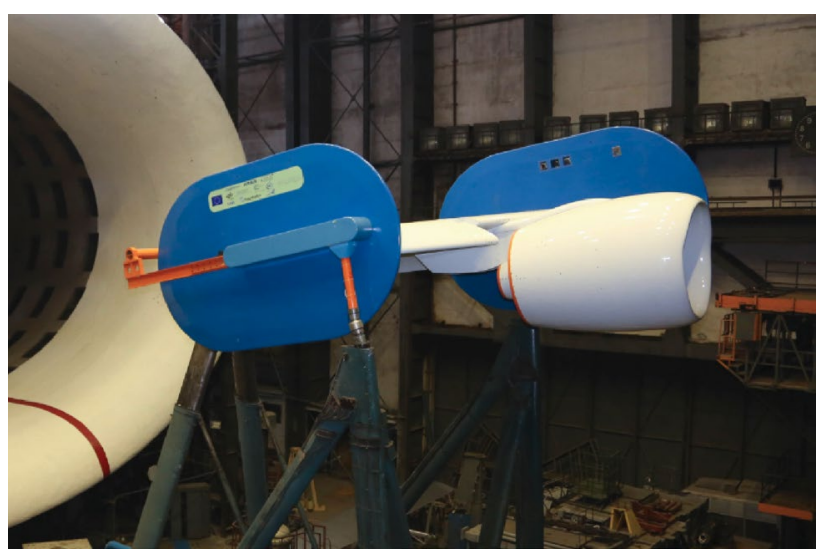

Fig. 1 LSM in TsAGI T-101 wind tunnel 


\subsection{Large scale test}

The LSM as described in the previous section was tested in the closed-loop subsonic wind tunnel T-101 at TsAGI. The elliptical cross-section of the nozzle outlet is $24 \times 14 \mathrm{~m}$, which indicates the dimensions of the open test section. Temperature and pressure correspond to environmental conditions. The facility allows Mach numbers up to 0.15 and Reynolds numbers based on the wing reference chord of up to $11.2 \times 10^{6}$. However, restrictions regarding recordable aerodynamic loads limit the inflow velocity to $48 \mathrm{~m} / \mathrm{s}$ leading to a Reynolds number of $10.75 \times 10^{6}$, and a Mach number of 0.144. Besides forces and moments measured via an external six-component underfloor mounted electricmechanical balance, pressure values were gathered using static pressure taps along three chordwise and two spanwise rows, indicated by the solid and dashed lines in Fig. 2. The area of separation prone flow and thus stall initiation was identified in several previous works, e.g. in [10,13], as the inboard area of the slat cut-out region, marked in Fig. 2. Since the focus of the present paper and the wind tunnel test in general lies on the evaluation of the flow field in this particular region, primarily the pressure values on the first spanwise and chordwise rows highlighted by dashed lines are used for validation purposes. Additionally, surface tuft visualization was employed in the region of interest to complement pressure measurements and give a more thorough insight in the separation topology. The orientation of the chordwise pressure tap rows perpendicular to the leading edge is a relic from aforementioned tests of the non-swept high-lift wing and is not based on any physical perceptions. The loads captured by the external balance include the forces acting on the model mounting and support, shown on the image in Fig. 1. Nevertheless, a meaningful evaluation of AFC effects using the lift coefficient $c_{\mathrm{L}}$ could be realized by examination of $\Delta c_{\mathrm{L}}$ values of tests with and without AFC

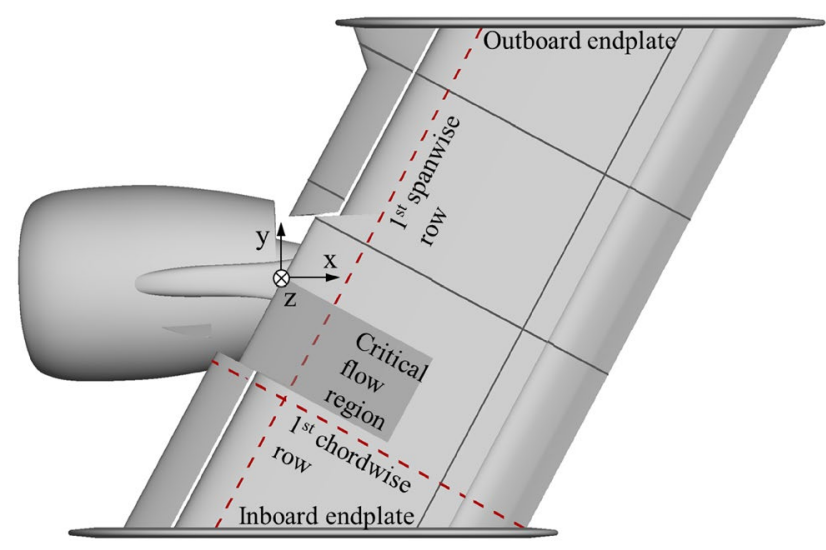

Fig. 2 LSM with pressure tap locations indicated by solid and dashed lines
[11]. The model mounting and the support were not modeled in the CFD simulations of the LSM.

\subsection{Small scale test}

The experiments on the SSM were performed in the KnappMeadow low speed wind tunnel at Tel-Aviv University. The wind tunnel is of Göttingen-type with atmospheric conditions inside the test section. The closed test section has a width of $0.61 \mathrm{~m}$, a height of $1.5 \mathrm{~m}$, and a length of $4.25 \mathrm{~m}$. The SSM is a downscale of approx. 1:8.4 with respect to the LSM. The endplates were replaced by rotatable plexiglas discs, flush mounted to the side walls of the test section with the purpose to maximize the SSM dimensions, see Fig. 3. A blockage factor, representing the ratio of the frontal model area to the test section's cross-sectional area, of $12.6 \%$ was detected at maximum measured inclination, which exceeds the general guideline for maximum correctable blockage of $10 \%$ [14]. No incidence angle corrections, solid blockage correction or any other type of wind tunnel corrections were applied to the measurement data. Static pressure data were recorded along the two spanwise and the two inboard located chordwise rows as shown for the LSM in Fig. 2. The outboard row was omitted due to space limitations for pressure tube placement. Furthermore, surface oil flow visualization and wake measurement data captured using a seven-hole pressure probe were acquired for validation of the CFD simulations. The 3D printed slat and flap limited the maximum inflow velocity to $25 \mathrm{~m} / \mathrm{s}$ due to material strength restrictions. Aluminum high-lift devices will be installed for future tests to allow an alignment of the Mach number to the LSM tests. The scaling factor and the reduced velocity lead

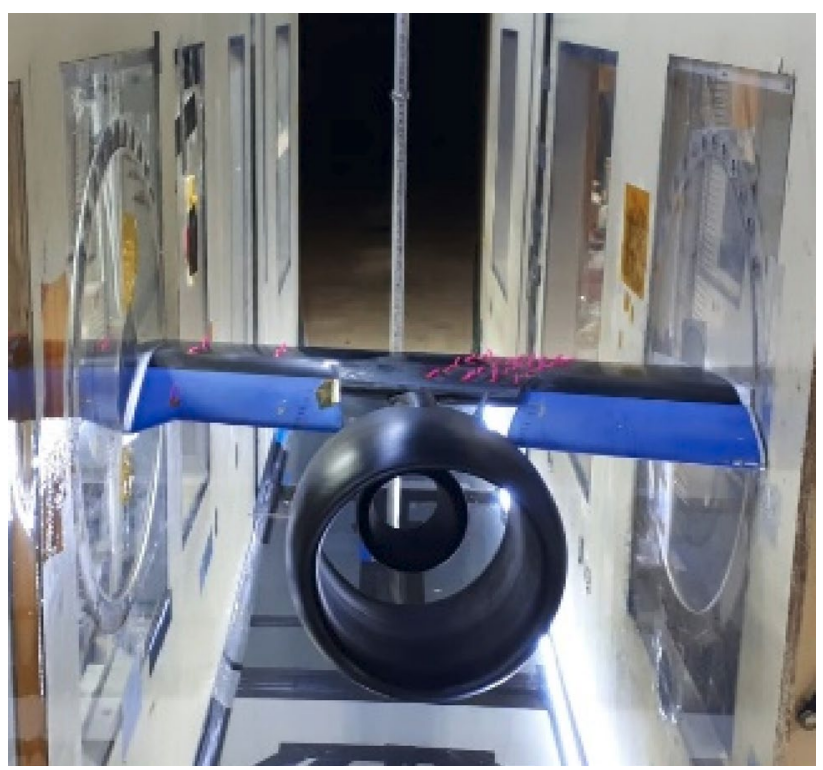

Fig. 3 SSM in Knapp-Meadow wind tunnel at Tel-Aviv University 
to a Reynolds number and a Mach number of about $0.66 \times$ $10^{6}$ and 0.075 , respectively. By contrast, the inflow velocity of the SSM CFD simulations was kept according to the LSM test conditions. The impact of the two-fold differences in Reynolds number and Mach number between the available SSM test data and the SSM simulations was part of separate studies within the current project and will be discussed in the course of this paper.

\section{Numerical approach}

In this section, the selected numerical setup will be described by introducing the flow solver with associated parameter settings, the boundary conditions, and the computational grid.

\subsection{Flow solver and parameter settings}

The simulations were conducted using the unstructured finite volume flow solver DLR TAU code [15] in steadystate Reynolds Averaged Navier-Stokes (RANS) mode. Unsteady RANS (URANS) simulations were employed in critical cases, where transient phenomena triggered significant oscillations in integral force coefficients. The computational grid was transformed into a cell-vertex dual cell grid in a preprocessing step. The three dimensional compressible RANS equations are solved with second order accuracy in time and space. A central scheme with scalar artificial dissipation was utilized for spatial discretization. Time integration was realized using an implicit Backward Euler method with lower-upper symmetric Gauss-Seidel (LUSGS) iterations. Pre-studies revealed that the best compromise between computational time, accuracy, and numerical stability could be achieved by closing the RANS equations with the Spalart-Allmaras Original (SAO) one-equation turbulence model (TM) [16]. To ensure comparability between the simulations of the SSM and LSM the TM version was not varied, besides the fact that an extension of the SAO TM for modeling the wind tunnel wall interference could be favorable [17]. Data from several studies, e.g. [18], have confirmed the TM version as a crucial "solution attractor" at angle of attacks where separation is predicted.

For acceleration of convergence, a multigrid approach with a $2 \mathrm{v}$ cycle and local time stepping was applied for all cases. Laminar to turbulent transition was neglected for both the SSM and the LSM by setting fully turbulent inflow conditions, as no significant laminar flow region was expected in the region of interest impinged by the highly vortical flow field even for the SSM. Dual time stepping was applied for time-accurate simulations. The time step size was selected in a way to guarantee 100 convective time steps per convective time $\left(t_{\text {conv }}\right)$, with $t_{\text {conv }}=c_{\text {ref }} / u_{\infty}$. About 300 inner iterations were required to achieve acceptable convergence for each intermediate steady-state computation.

\subsection{Computational grid}

The hybrid grids were created in Pointwise V18.0 R3 [19]. Quad-dominant elements were used to discretize the wing surface. Hexahedral and prismatic elements were generated by extruding the surface mesh in the wall-normal direction. The initial cell height was chosen to guarantee a non-dimensional wall distance $y^{+}$of less than one making it suitable for the SAO TM. A growth rate of 1.2 was chosen for boundary layer mesh according to best practice gridding guidelines [20]. This results in a total number of quasi-structured layers between 40 and 45 on both the model and the sidewalls required for sufficient boundary layer resolution.

Special care was taken for the grid in the slat cut-out area to ensure an adequate resolution of the vortices emanating from the strake, the nacelle, and the exposed corners and edges of the slat. Nearly isotropic hexahedral grid blocks were created on top of the boundary layer mesh, as shown in Fig. 4. Prior numerical studies, e.g. [13, 21] have noted that these vortices play an important role in the initiation of flow separation when interacting with the attenuated boundary layer flow. In addition, the cove region in between the high-lift elements and the main wing were resolved with structured grid elements according to previous findings [22], see bottom sectional-views in Fig. 4. Local surface mesh refinement toward concave corner regions, like the modelendplate junction for the LSM or model-tunnel wall junction for the SSM, was realized to minimize chopped cell regions. In case of the LSM the remaining farfield, with an extension of about $50 \times c_{\text {ref }}$ in all directions of space, was filled with unstructured pyramids and tetrahedra. The inflow conditions were imposed on the farfield boundary for the LSM simulations. The wind tunnel enclosure of the SSM was modeled by the test section walls. The wind tunnel wall boundary conditions were set to viscous for the sidewalls and inviscid for the ceiling and the floor, as no direct flow interaction was expected with the latter and the boundary layer thickness on the test section walls is rather small compared to its crosssectional area. A pressure coupling procedure was selected, where the pressure at the test section outlet was changed in an iterative manner to reach the inflow conditions at a defined measurement coordinate for the converged solution. To incorporate the SSM inside the wind tunnel and at the same time allow a rotation about the wind tunnel transverse axis for the $\alpha$-sweep computation, the Chimera grid overlapping technique was utilized. The Cartesian wind tunnel grid comprising a hole-cutting, is assembled with the SSM mesh in a way to allow a sufficient overlapping for interpolation, see Fig. 5. The final grids contained about 40 million grid points in total. The SSM grid was derived from the LSM 

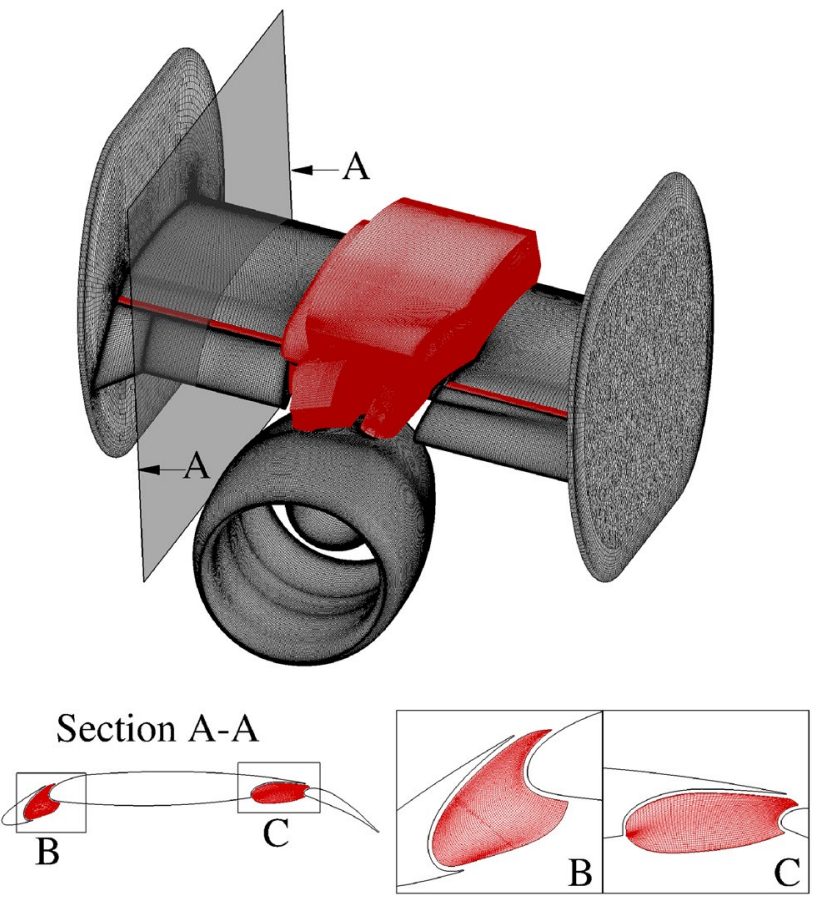

Fig. 4 LSM surface mesh with hexahedral mesh blocks in the slat cut-out and the cove region shown in red

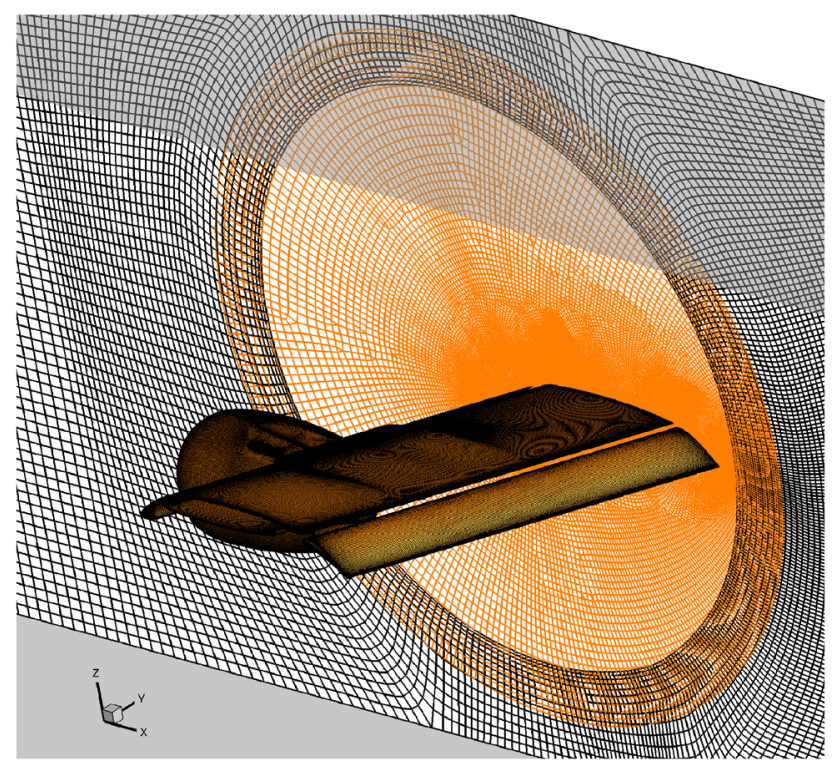

Fig. 5 SSM grid (orange) with Chimera zone mounted inside the Meadow wind tunnel test section (black)

grid by adapting the boundary layer mesh according to the smaller Reynolds number. The grid for the LSM at modified Reynolds number (MR-LSM) corresponding to the SSM tests was derived by simply scaling the LSM mesh down to SSM scale. Even though the relative boundary layer thickness for the MR-LSM is expected to be larger compared to the LSM, an acceptable resolution of the boundary layer could be assured, due to the high extension of the boundary layer grid for the LSM. As expected, the $y^{+}$value of the first cell height is far smaller than one for the MR-LSM grid, despite the fact that $y^{+}$does not directly correlate with the Reynolds number.

\section{Results}

Before conducting the cross-comparison of the three sets of simulation for the evaluation of the Reynolds number and wind tunnel wall effects, a validation against experimental data is indispensable. A large amount of measurement data was acquired from the TsAGI and Knapp-Meadow wind tunnel tests. In order not to go beyond the scope of this work, only selected data representing both cases-those with attached flow and those with flow separation in the slat cutout region-are presented in the following.

\subsection{Validation of numerical simulations}

\subsubsection{TsAGI wind tunnel measurements}

As mentioned in Sect. 2.2, the model mounting was not excluded from force measurements. Owing to the fact that neither the mounting struts nor the open-jet wind tunnel environment were modeled in the CFD simulations a direct comparison of force coefficients is difficult. Neglecting the open-jet may cause deviations in the effective incidence angle, streamline curvature, and drag force due to blockage effects and horizontal buoyancy [23]. As the individual impact of these effects was not evaluated in detail during the test campaign they pose a potential source of inconsistency toward CFD and should, therefore, be kept in mind. The stall characteristic mirrored in the lift coefficients do not exhibit a significant influence from the model mounting. Thus, a qualitative comparison with CFD is appropriate. The lift and drag curves are illustrated in Fig. 6 for both CFD and experiment. The error bars added to the simulation results represent the maximum amplitudes of prevailing fluctuations caused by unsteady phenomena related to flow separation. The focus of the subsequent evaluation lies on the $c_{\mathrm{L}}-\alpha$ curve, the $c_{\mathrm{D}}-\alpha$ curve is plotted for the sake of completeness. A pure assessment of the experimental $c_{\mathrm{L}}-\alpha$ curve demonstrates the specific character of the high-lift model, which does only qualitatively resemble the character of an aircraft in landing configuration. Comparably high angles of attacks were obtained without stall onset visible in the $c_{\mathrm{L}}-\alpha$ slope. This unexpected stall behavior is attributable to the research model's peculiarities described in Sect. 2.1. The simulation results show an offset from the experimental data which is on the 


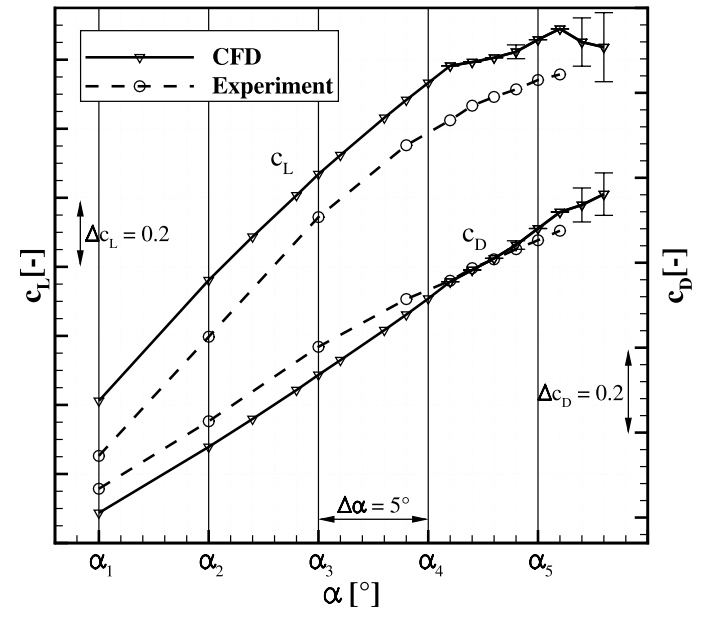

Fig. 6 Lift and drag curve from CFD and TsAGI measurements, $R e=10.75 \times 10^{6}$ and $M=0.144$

order of $\Delta c_{\mathrm{L}}=0.1-0.15$. The declination in the predicted $c_{\mathrm{L}}-\alpha$ slope at $\alpha_{4}+1^{\circ}$ observed for the simulations is not visible in the experiments, despite the close clustering of measurements at this critical range of incidence angles. The initial goal to increase $\alpha$ until clear degradation of $c_{\mathrm{L}}$ appears as a sign of large scale flow separation could not be realized due to balance recording constraints. The incidence range was extended about $2^{\circ}$ for the simulations, which revealed that a decrease in the $c_{\mathrm{L}}-\alpha$ slope is expected for $\alpha>\alpha_{5}+1^{\circ}$. No strong increase in $c_{\mathrm{D}}$ can be observed for this $\alpha$ range which is usually associated with stall. Despite the limited $\alpha$ range, the separation area in the slat cut-out region was proven to be large enough for the experiment to be effectively evaluated regarding its controllability using AFC even for $\alpha<\alpha_{5}+1$.

Further potential reasons for the deviations in the $c_{\mathrm{L}}-\alpha$ curve between CFD and experiment include an insufficient mesh resolution in areas, which were not of particular interest in this project but may pose a significant contribution to the overall force coefficients. Examples of such areas are the nacelle wake below the pressure side of the wing and the intersection area between the wing and the endplates. Additionally, individual incidence measurements via inclinometers on the wing and the nacelle revealed maximum deflections of up to $1.7^{\circ}$ in $\alpha$ due to a deformation of the heavy wind tunnel model.

A comparison of the $c_{\mathrm{p}}$-distribution along the first spanwise and chordwise rows marked in Fig. 2 is shown in Fig. 7. The subfigures on the left show the corresponding results at pre-stall $\alpha=\alpha_{1}$ and the subfigures on the right at post-stall $\alpha=\alpha_{4}+2^{\circ}$. Overall, a good agreement is visible on the pressure side along the chordwise line for all three wing elements. On the suction side, the CFD results predict more pronounced suction peaks on the flap's leading edges for both presented $\alpha$ and on the wing leading edge for $\alpha=\alpha_{4}+2^{\circ}$. Keeping in mind the large model deformation at high $\alpha$, these moderate deviations are acceptable.

The spanwise $c_{\mathrm{p}}$ distributions show a good qualitative match as well except for some outliers. The high-pressure plateau extending from approx. $y_{\text {norm }}=-0.2-0.1$ represents the wing section downstream from the slat cut-out. This higher $c_{\mathrm{p}}$ level results from the missing circulation effect of the slat which is usually responsible for a reduction of the pressure peak on the wing's leading edge. This section is characterized by strong spanwise pressure variations for
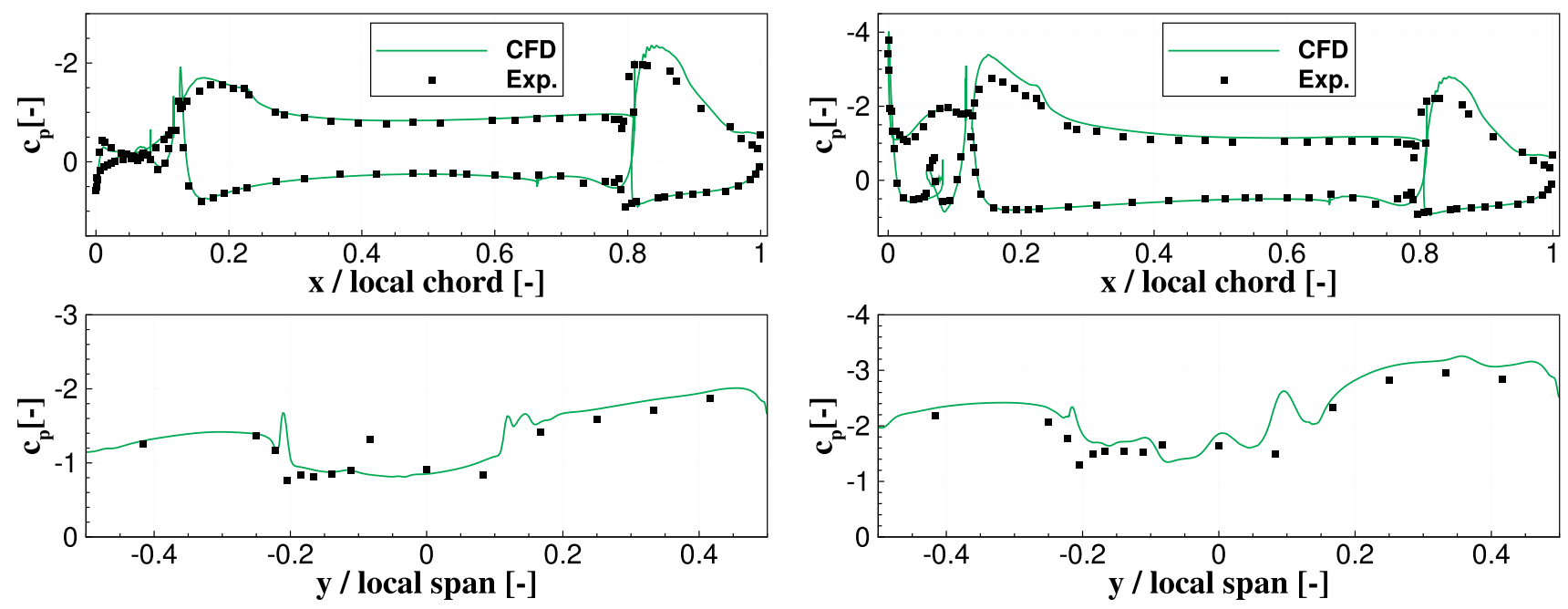

(a) $\alpha=\alpha_{1}$

(b) $\alpha=\alpha_{4}+2^{\circ}$

Fig. 7 Chordwise and spanwise $c_{\mathrm{p}}$-distribution on the LSM along the paths marked in Fig. 2 
larger $\alpha$, even on the outboard side $y>0$ of the slat cut-out, where no flow separation was evident. Due to the small number of pressure taps in this area, these spatial pressure fluctuations can not be reflected by the measurements. The simulations revealed a correlation between $\alpha$ and the vorticity in the slat cut-out region. The vortices are less pronounced at small $\alpha$ and thus induce less vortex-boundary layer interaction. As long as no large separation areas are developed, this interaction can be directly identified in the $c_{\mathrm{p}}$ values. Thus, this characteristic of $c_{\mathrm{p}}$ variations represents a potential indicator for stall onset, which can be used for closed loop AFC. The discrepancy between CFD and experiment downstream from the outboard slat edge at about $y_{\text {norm }}=0.2 \mathrm{can}$ be attributed to small inconsistencies between the geometry used in the simulations and the geometry used in the experiments. While the CFD model comprised a slight spanwise gap between the main wing and the outboard slat segment, this gap was not part of the tested geometry, see red circled areas in Fig. 8.

Surface flow patterns in the outboard slat cut-out area were visualized using luminescent mini-tufts with high spatial resolution. The bottom line in Fig. 8 shows integral tuft images at $\alpha=\alpha_{1}, \alpha=\alpha_{4}+2$ and $\alpha=\alpha_{5}+1$. It is clearly evident that at $\alpha=\alpha_{1}$ no deviation from inflow velocity direction is present, which indicates fully attached flow. The tuft images at $\alpha=\alpha_{4}+2$ and $\alpha=\alpha_{5}+1$ show extended zones with vibrating tufts. The flow separation areas marked by dashed lines in Fig. 8 were extracted in a postprocessing step by Schlösser et al. [12]. The corresponding CFD results are shown in the top row of Fig. 8. The contour of the $x$-component of the friction coefficient $c_{\mathrm{f} x}$ is illustrated together with surface streamlines. Areas with negative $c_{\mathrm{f} x}$ values, indicating reversed flow and thus flow separation, are highlighted in blue. When comparing to the tuft images a good agreement in both the location and the size of the separation area can be proven, which encourages the subsequent investigations in Sect. 4.2.

\subsubsection{Knapp-Meadow wind tunnel measurements}

In case of the small scale tests, a lower $\alpha$ range compared to the large scale test is investigated. The reason for this is explained in Sect. 4.2. No balance measurements were conducted and the two chordwise and two spanwise rows of pressure taps are not sufficient for a proper force coefficient integration representative for the test model. Hence, the pressure measurements represent the primary source for validation of the SSM CFD results. The $c_{\mathrm{p}}$ distributions, again along the spanwise and chordwise lines highlighted in Fig. 2, at $\alpha=\alpha_{1}-10^{\circ}, \alpha=\alpha_{1}$ and $\alpha=\alpha_{2}$ are presented

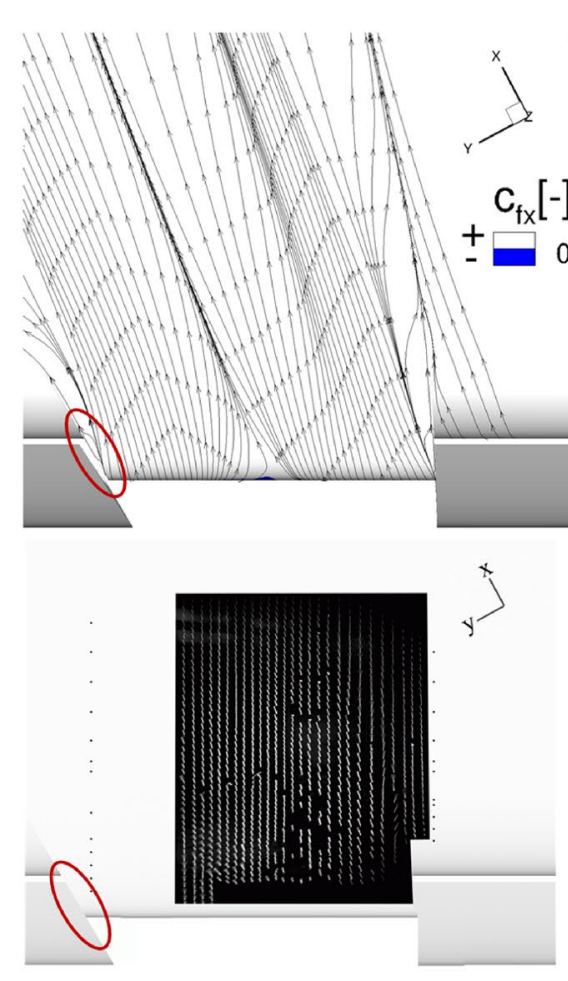

(a) $\alpha=\alpha_{1}$

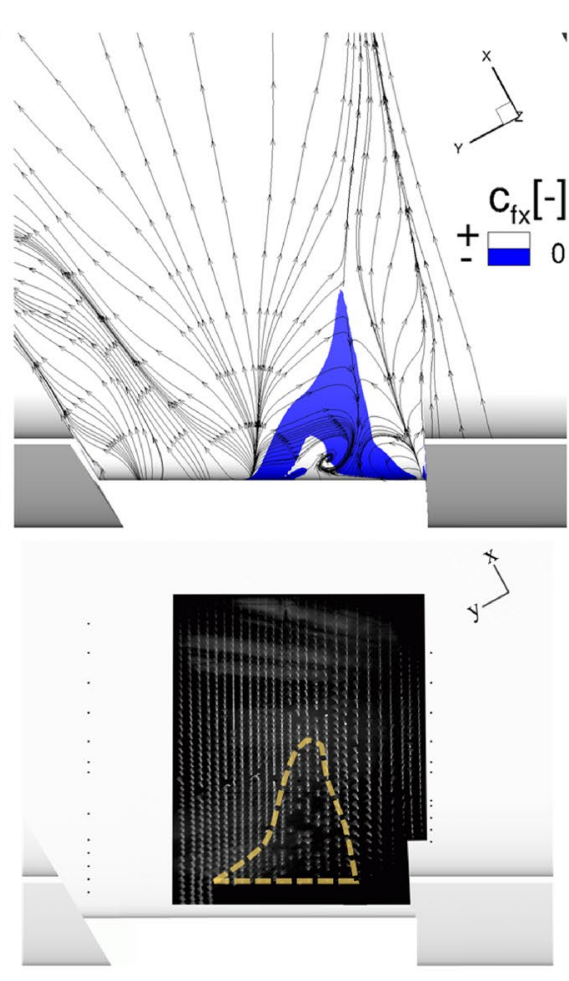

(b) $\alpha=\alpha_{4}+2^{\circ}$

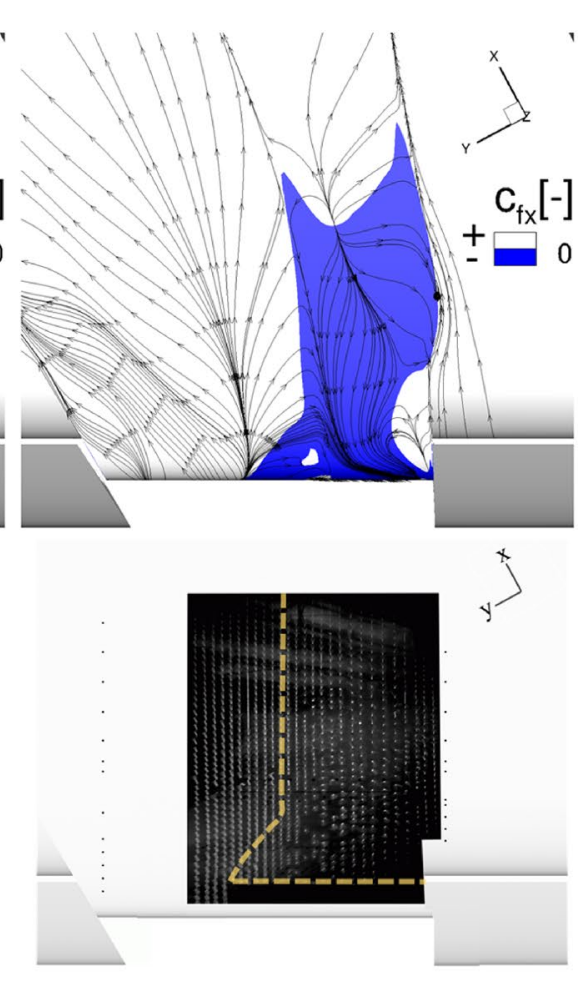

(c) $\alpha=\alpha_{5}+1^{\circ}$

Fig. 8 Separation area in CFD and experiments. Negative $c_{\mathrm{fx}}$ indicates reversed flow in simulations and framed area on tuft images indicates reversed flow in experiments 
in Fig. 9. Besides the CFD results obtained with the DLR TAU code, the results obtained with the unstructured CFD code Edge [24] are depicted. The Edge code was used for the separate Mach number and Reynolds number independence study explained in Sect. 2.3.

A similar computational grid and similar numerical settings were used in terms of turbulence model and numerical dissipation to justify the comparison with the TAU code results. Occurrence of corner separation on the outboard wing-sidewall junction represents one major issue for the SSM tests inside the closed test section environment. Unlike as for the LSM tests, the leading edge extension was not a sufficient measure to prevent corner separation. The wall normal velocity gradient of the boundary layer on the test section wall at the vicinity of the SSM is small when compared to the respective gradient on the endplates of the LSM due to boundary layer development along the test section path. The CFD simulations using the TAU code revealed a strong influence of the computation procedure on the onset of corner separation. In the first approach, the simulations at a particular $\alpha$ were started from scratch and in the second approach a "real" $\alpha$-sweep computation was conducted. For the $\alpha$-sweep computation the $\alpha$-range was traversed in a consecutive manner. The $c_{\mathrm{p}}$ plots contain both CFD results obtained with the TAU code. No Edge code simulations were conducted for the smallest computed incidence angle $\alpha=\alpha_{1}-10^{\circ}$. The SSM-flap was not instrumented with pressure taps on the evaluated chordwise row. The simulation results at $\alpha=\alpha_{1}-10^{\circ}$ show a good match with experimental data, except for some outliers. The same applies to the results at $\alpha=\alpha_{1}$ and $\alpha=\alpha_{2}$, with the exception for the simulation results obtained with TAU using the first approach at $\alpha=\alpha_{1}$ and the Edge code at $\alpha=\alpha_{2}$. The latter mentioned simulations reveal large discrepancies to the measurements due to the erroneous prediction of corner separation. TAU CFD results conducted for $\alpha=\alpha_{1}$ without employing results at smaller $\alpha$ show a large area of corner separation, which is not evident in the measurements. This behavior is reflected in the spanwise pressure distribution, where the outboard corner, affected by corner separation, is located in the positive $y$-coordinate range. The Edge code is rather dissipative regarding corner flow separation and thus even at $\alpha=\alpha_{2}$ a high flow acceleration level can be observed in the outboard region of the spanwise $c_{\mathrm{p}}$-distribution which is not conform to the measurements. Despite the large spanwise extension of corner separation, which can be estimated from the spanwise $c_{\mathrm{p}}$-distribution at $\alpha=\alpha_{2}$, the impact on the $c_{\mathrm{p}}$ -distribution in the slat cut-out area is low, which encourages the evaluation of AFC in this region without the necessity to derive measures for prevention of corner separation. The generally small differences in Edge code results obtained at the same incidence angles confirm the limited influence of

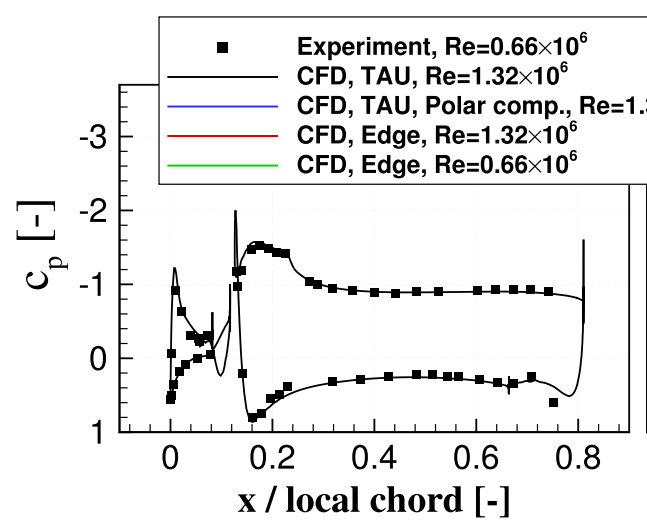

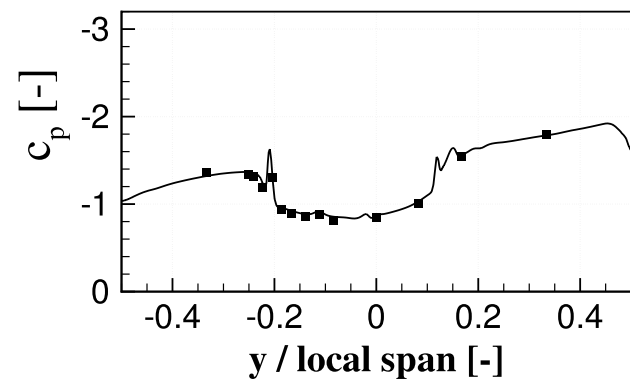

(a) $\alpha=\alpha_{1}-10^{\circ}$

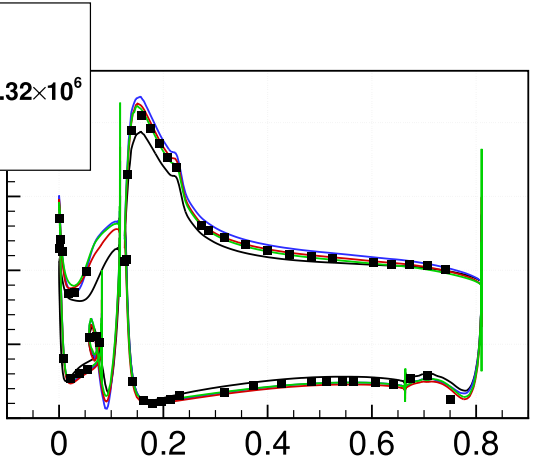

$\mathrm{x} /$ local chord [-]

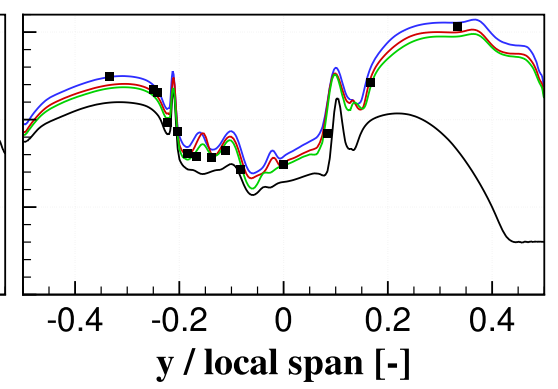

(b) $\alpha=\alpha_{1}$

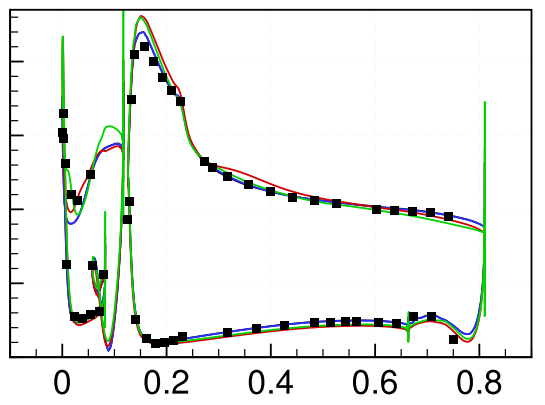

$\mathrm{x} /$ local chord [-]

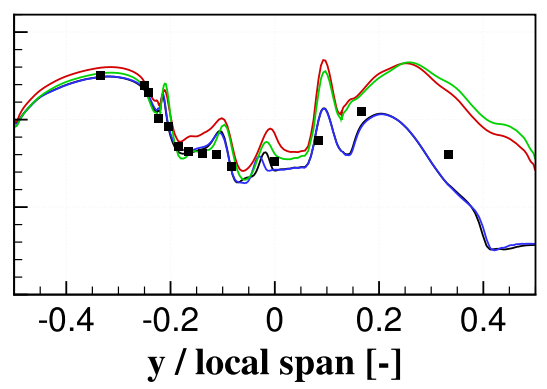

(c) $\alpha=\alpha_{2}$

Fig. 9 SSM $c_{\mathrm{p}}$-distributions along the inboard chordwise row and the front spanwise row shown in Fig. 2 
Reynolds number and Mach number effects for the SSM test. These results are consistent with those of recent experimental studies conducted for the LSM [12] and suggest that the flow around the investigated geometry is largely Reynolds number and Mach number independent within the investigated range. Furthermore, the good agreement in TAU code and Edge code results approve solver independency despite the investigated complex flow field.

Since only one static pressure tap was placed on the outboard wing along the front spanwise measurement line a complementary confirmation method for the onset of corner separation was utilized.

Wake measurements, as shown in Fig. 10a for $\alpha=\alpha_{1}+2$ were conducted in the $y z$-plane about $1.6 \mathrm{~m}$ downstream from the coordinate origin depicted in Fig. 2. The corresponding TAU code results at the same $\alpha$ are depicted in Fig. 10b, c. The subfigures Fig. 10b, c illustrate the results obtained by the $\alpha$-sweep computation and the computation starting from scratch, respectively. Since no special effort was put into resolving the computational grid in the model wake region, the analysis undertaken here is of qualitative nature. A downstream tracing of vortices emanating from the SSM reveals that the most dominant vortices captured by the measurement plane are those of the nacelle. Other areas with low streamwise velocity show vortices emerging at the strake, the pylon, the slat ends and the sidewall-wing junction. The simulation from scratch predict large scale corner separation at $\alpha=\alpha_{1}+2$, which is not evident in the $\alpha$ -sweep simulation result. This feature is also reflected in the respective wake images. The simulation results in Fig. 10c show the wake of the corner separation in the outboard area at $y>0$, which is clearly dominant and leads to a dissipation of the nearby vortices. The measurements clearly reflect the results of the $\alpha$-sweep simulations without corner separation and thus verify the statements above. Based on this finding, the CFD results of the SSM shown in the following represent those extracted from the $\alpha$-sweep simulations.

The last validation procedure of the SSM simulations covers the surface flow patterns in the slat cut-out region. Oil flow visualizations are available for several $\alpha$.

A good match between CFD and experiments in the orientation of surface streamlines, separation onset, and separation size could be shown and is exemplary demonstrated in Fig. 11 for $\alpha=\alpha_{2}-1^{\circ}$. The CFD results include surface contour values of $c_{\mathrm{f} x}$ and the surface stream lines. Especially, the similarity regarding converging streamlines in the slat cut-out area is clearly evident.

The thorough validation of the CFD results in this section which demonstrated a highly satisfactory agreement with experimental data, represents a proper foundation for the evaluation and cross-comparison of simulation results in the following section.

\subsection{Reynolds number and wind tunnel wall effects}

The flow around geometries mounted in subsonic atmospheric wind tunnels with a closed test section may be manipulated due to effects related to solid blockage, wake blockage, model mounting, leakage flow, and induced incidence angles [25]. Scaling effects related to different Reynolds numbers were extensively investigated for high-lift wings with different degrees of complexity by Rudnik and Germain [26]. Swept high-lift configurations with slotted design are subject to secondary flow effects induced by the boundary layers of upstream elements and the flow emanating from the cove area, which affects their Reynolds number dependency compared to those without high-lift devices. Wings in general experience Reynolds number effects which are related to laminar-turbulent transition, bursting of laminar bubbles and boundary layer thickness. Due to the complex flow topology
Fig. 10 Vertical wake-plane downstream from the SSM with streamwise velocity contour at $x \approx 1.6 \mathrm{~m}$ and $\alpha=\alpha_{1}+2^{\circ}$ $\left(R e_{\text {exp. }}=0.66 \times 10^{6}\right.$ and $\left.R e_{\mathrm{CFD}}=1.32 \times 10^{6}\right)$. Black frames mark the measurement area

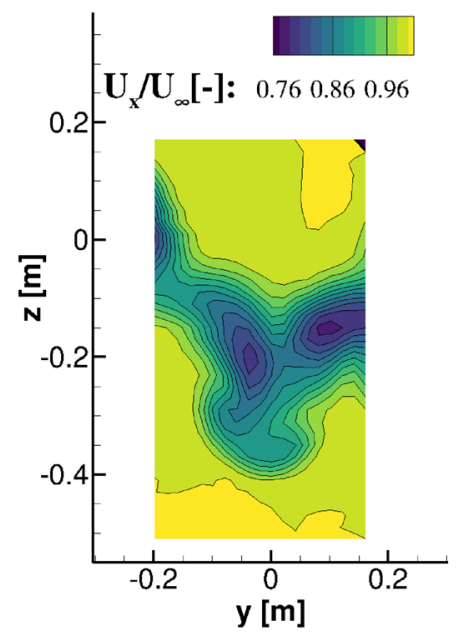

(a) Experiment

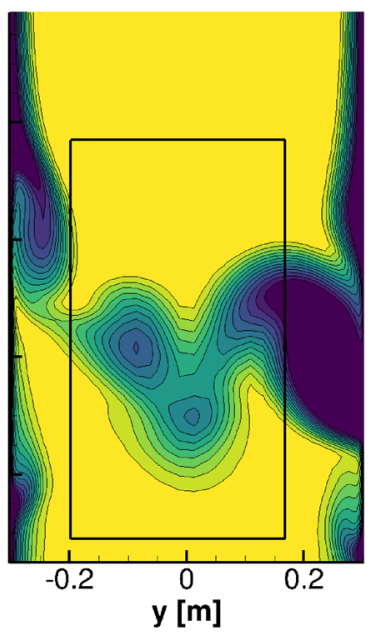

(b) CFD $\alpha$-sweep

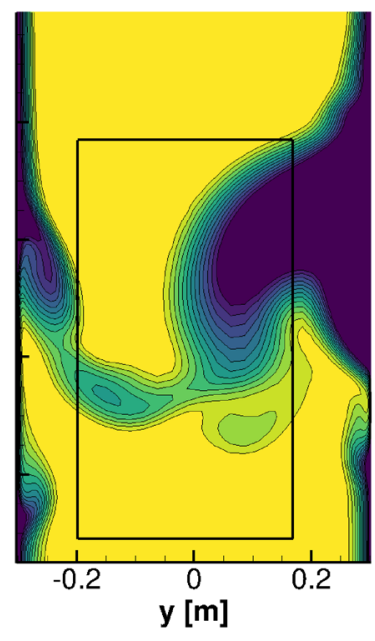

(c) CFD Scratch 
Fig. 11 Comparison of surface streamline patterns on the SSM at $\alpha=\alpha_{2}+1^{\circ}$, $R e_{\text {exp. }}=0.66 \times 10^{6}$ and $R e_{\mathrm{CFD}}=1.32 \times 10^{6}$

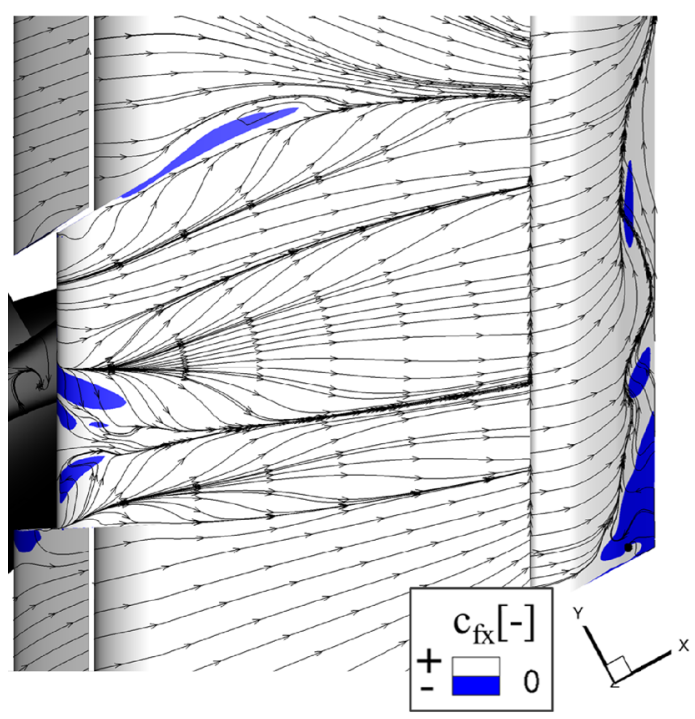

(a) CFD

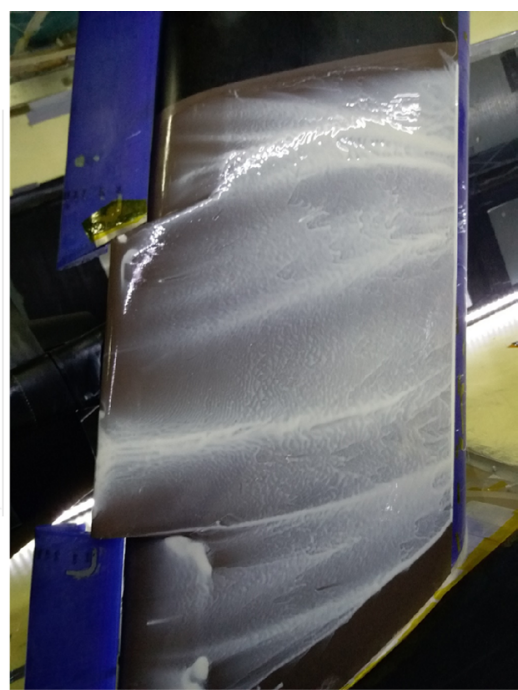

(b) Experiment on high-lift wings only a small number of trends associated with scaling effects were derived until today, which include the increase in maximum lift and decrease in total drag with increasing Reynolds number $[3,26]$. Nevertheless, the generalizability even for these trends is not valid as other studies have revealed totally opposite results [9].

The focus of the present study is to assess the Reynolds number and wind tunnel wall effects and the way these factors impact the data extrapolation from small scale tests in closed test section environments to large scale tests in open test section environments. As proven in Sects. 4.1.1 and 4.1.2, large scale separation occurs in the slat cut-out area even without the typical stall characteristics of a sudden lift drop. In addition, the drag force is of minor importance in the scope of this project. Therefore, neither the $c_{\mathrm{L}, \max }$ nor $c_{\mathrm{D}}$ are part of the following evaluation.

The lift curves extracted from the simulations of the LSM, the MR-LSM, and the SSM are shown in Fig. 12. As the SSM does not carry endplates which are exposed to the flow, the $c_{\mathrm{L}}$ values for all presented models were derived by neglecting the endplates during the force integration. The $c_{\mathrm{L}}$ curve of the LSM reveals a nearly constant shift of approx. $\Delta c_{\mathrm{L}}=0.05-0.1$ to the $c_{\mathrm{L}}$ curve of the MR-LSM up to the maximum evaluated $\alpha$. This difference in $c_{\mathrm{L}}$ values might be attributable to first-order scaling effects caused by increasing displacement thickness with decreasing Reynolds number. However, the Reynolds number effect on $c_{\mathrm{L}}$ values in the linear lift range for high-lift configurations cannot be simply reduced to the effect related to the boundary layer displacement thickness, as aforementioned experimental studies by Rudnik et al. [26] demonstrated that there is no clear correlation between Reynolds number and $c_{\mathrm{L}}$ for high-lift configurations with realistic geometric complexities.

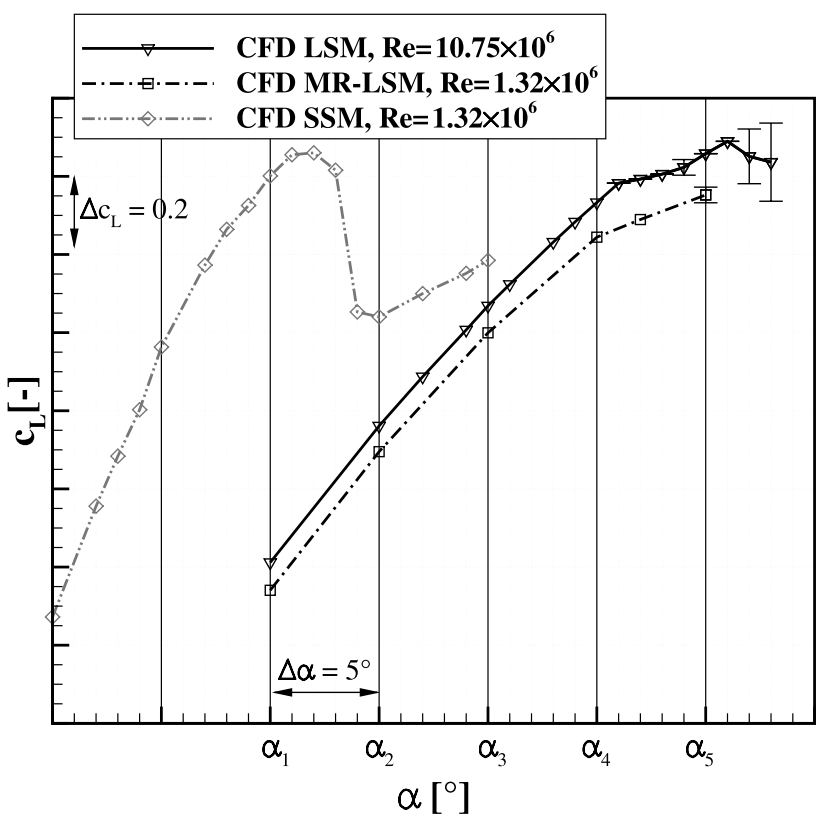

Fig. 12 Comparison of lift curves for LSM, SSM, and MR-LSM

When comparing the $c_{\mathrm{L}}$ curve of the SSM with the MR-LSM, the pure effect of the wind tunnel walls can be extracted, which is of a higher order of magnitude compared to the Reynolds number effect. The linear lift range shows neither a similarity in the slope nor in the integral values. A substantial increase of about $\Delta c_{\mathrm{L}}=1.05$ at $\alpha=\alpha_{1}$ is apparent. The sudden drop in $c_{\mathrm{L}}$ at $\alpha=\alpha_{1}+3^{\circ}$ for the MR-LSM can be ascribed to the large scale corner separation in the outboard area instead of stall onset in the slat cut-out region. Differences in $c_{\mathrm{L}}$ values in the linear range $\alpha<\alpha_{1}+2^{\circ}$ are attributable to the blockage effect. 
The difference in the $c_{\mathrm{L}}$ slopes is related to the increase of the blockage factor with growing $\alpha$. As soon as the incidence of the SSM exceeds $\alpha=\alpha_{3}$ the simulations expose oscillations in the force coefficients, which originate from nacelle separations and leading-edge stall. These oscillations show no periodic character usually required for time averaging. Thus, the corresponding $c_{\mathrm{L}}$ values of the SSM are not incorporated in Fig. 12. The analysis of simulation results in this section will demonstrate that despite the enormous deviations in the lift curves, especially between the MR-LSM and the SSM, certain adjustments based on the evaluation of $c_{\mathrm{L}}$ values allow to derive a similar flow field in the slat cut-out area.

A detailed flow field analysis of all three sets of simulations was conducted to derive accurate measures for the SSM tests required for flow adjustment toward the LSM tests. The results reveal that the flow fields around the LSM and the SSM show highly satisfactory agreement when considered at the same $c_{\mathrm{L}}$. This method only applies to the linear lift regime. Thus, the SSM shows the same flow features in terms of local acceleration and flow separation at significantly smaller $\alpha$. However, due to the difference in the slope of the $c_{\mathrm{L}}$ curves a simple $\alpha$-shift is insufficient. To prove this characteristic the following evaluation of CFD results exemplary compares data from the LSM at $\alpha=\alpha_{3}$ with data from the SST at $\alpha=\alpha_{1}-5^{\circ}$. Besides the LSM and SSM results at adjusted $\alpha$, the results from the MR-LSM and the SSM at unadjusted $\alpha$ are used for the analysis. The selection of $\alpha=\alpha_{3}$ for the LSM evaluation is based on the fact that separation initiation in the slat cut-out was detected at this $\alpha$. A proof of flow similarity between the SSM and the LSM at this particular point is an essential step for the subsequent work within this project.

Figure 13 shows the $c_{\mathrm{p}}$ contour levels and negative $c_{\mathrm{f} x}$ regions with surface streamlines on the suction side of the model for all cases described above. As already predicted by the $c_{\mathrm{L}}$ curve, differences in separation area, surface streamlines, and pressure distribution between the LSM and the MR-LSM are barely visible. The SSM at the same $\alpha$ shows extended regions of flow separation at the inboard slat cutout region and at the outboard model-wing junction, which have a huge effect on the $c_{\mathrm{p}}$-distribution. However, when reducing the incidence of the SSM toward the $\alpha$ where the $c_{\mathrm{L}}$ of the SSM matches the $c_{\mathrm{L}}$ of the LSM, the similarity in surface flow patterns, $c_{\mathrm{p}}$-distributions, and separation areas can be verified, see Fig. 13a, d.

For a more quantitative comparison, the $c_{\mathrm{p}}$-distributions of the main wing along the first chordwise line marked in Fig. 2 are plotted in Fig. 14. Besides the results at $\alpha=\alpha_{3}$, the results at $\alpha=\alpha_{2}$ are added to demonstrate the general validity of the $\alpha$-adaptation approach. The SSM results corresponding to the LSM results at $\alpha=\alpha_{2}$ were extracted at $\alpha=\alpha_{1}-7^{\circ}$ to obtain similarity in $c_{\mathrm{L}}$, see Fig. 12 . The data of the SSM at unadjusted $\alpha$ is omitted for the sake of clarity and due to the strong dissimilarity already proven in the contour plots in Fig. 13.

The difference in the Reynolds number between the LSM and the MR-LSM mainly affects the suction peak. The difference between the LSM and the SSM at adjusted $\alpha$ is smaller at the suction peak, but somewhat larger along the center region and the rear part of the wing. Yet, the overall differences are small and within a range where potential AFC
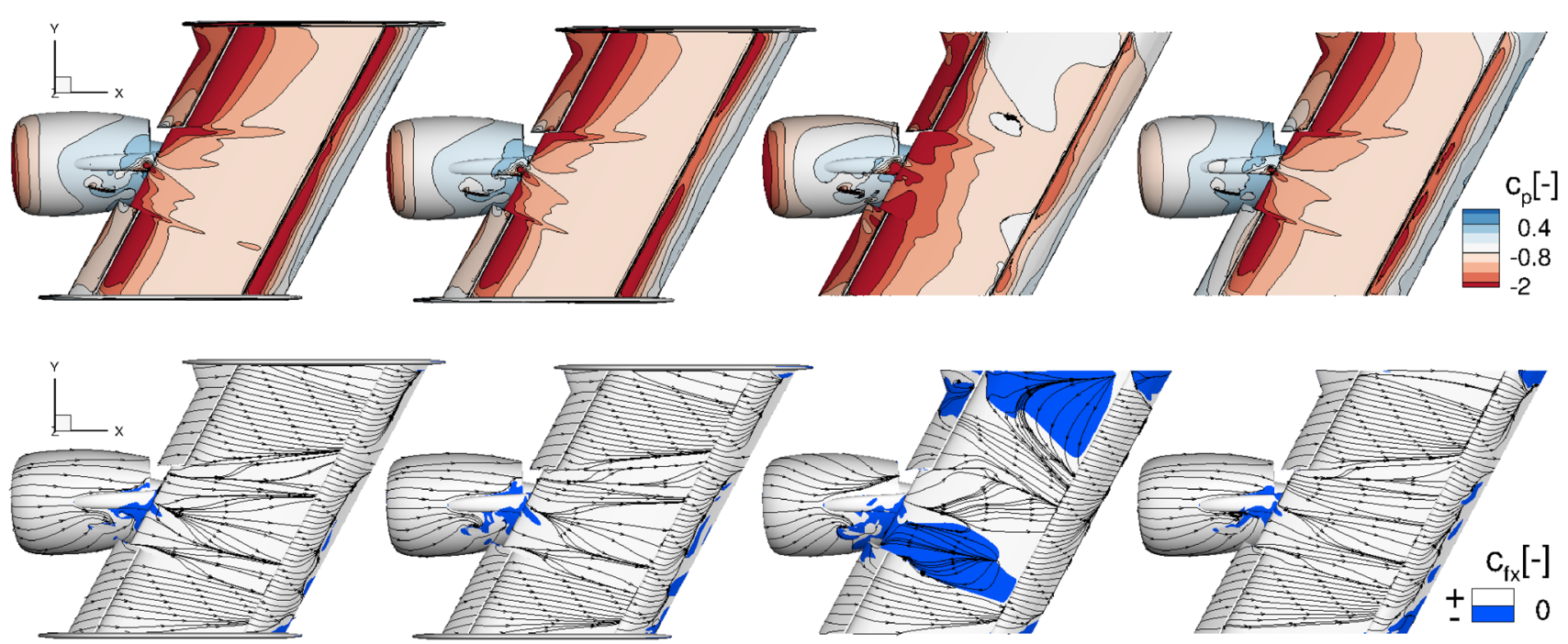
(a) LSM at $\alpha=\alpha_{3}$
(b) MR-LSM at $\alpha=\alpha_{3}$
(c) SSM at $\alpha=\alpha_{3}$
(d) SSM at $\alpha=\alpha_{1}-5^{\circ}$

Fig. $13 c_{\mathrm{p}}$ contour and $c_{\mathrm{f} x}$ contour with surface streamlines at $\alpha=\alpha_{3}$ resp. $\alpha=\alpha_{1}-5^{\circ}$ 
Fig. 14 Pressure distribution on main wing for the LSM, the SSM with adjusted $\alpha$ and the MR-LSM

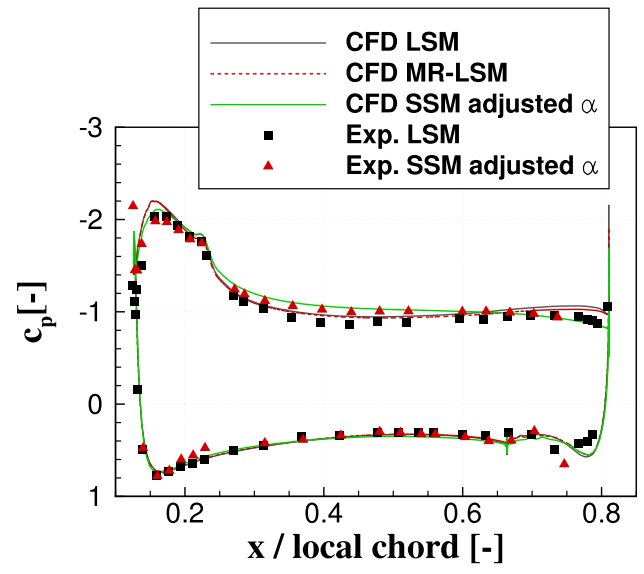

(a) $\alpha=\alpha_{2}$

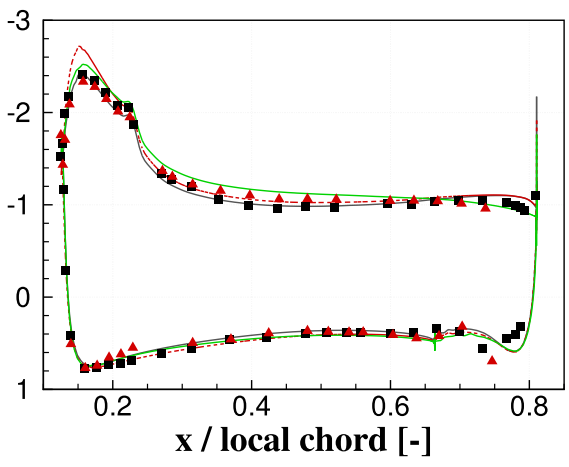

(b) $\alpha=\alpha_{3}$ effects should be visible. Furthermore, measurement data of the LSM and the SSM at adjusted $\alpha$ is added to the CFD results shown in Fig. 14 to underline the outcome derived from the simulations.

As already emphasized in the course of this paper, the vortical flow field in the extended slat cut-out area is one of the primary causes leading to premature flow separation. Therefore, an individual evaluation of the vortex structures in the slat cut-out region is of major importance. The vortex structures in the slat cut-out area of the LSM were identified using the $\lambda_{2}$ vortex-core visualization criteria. The results show that all eight dominating vortices comply with those introduced in $[13,27]$ for similar configurations, both in rotation direction and location. The vortices in the slat cutout region of the LSM are visualized in Fig. 15, top left. A qualitative agreement in vortical structures can be derived between the LSM, the MR-LSM (Fig. 15, top right) and the SSM at adjusted $\alpha$ (Fig. 15, bottom left). Again, the SSM at unadjusted $\alpha$ (Fig. 15, bottom right) reveals huge differences to the LSM. The already predominant flow separation in the inboard slat cut-out region leads to a displacement of the strake vortex while the other vortices on the inboard half of the slat cut-out region, which play a vital role in initiating flow separation, are dissipated. Since previous studies have demonstrated that the vortex path, and thus the effectiveness of vortex generators in the form of forebody strakes [28] and nacelle strakes [26], is strongly dependent on the Reynolds number, a more quantitative comparison of vortex paths in the slat cut-out region with regard to their Reynolds number and wind tunnel wall influences is required. An exact identification of vortex core locations of the dominant vortices in the slat cut-out region is not feasible due to strong vortex interaction and the related vortex merging. Only the strake vortex, the outboard leading edge step vortex and the pylon shoulder vortex witness less interaction with nearby vortices and can be used for vortex core detection. However, the latter two vortices are located in the outboard slat cut-out region and the strake vortex is further detached from the surface for the investigated configuration. Thus, these vortices do not directly affect the inboard separation area. Nevertheless, to provide a deeper insight in the vortical flow and its interaction with the unprotected wing downstream from the nacelle, the vortices are visualized in vertical planes parallel to the wing's leading edge using vorticity contours, see Fig. 16. The volume streamlines in the vicinity of the wing surface were added to track their path, which allows to assign them to the upstream geometric components. The LSM, the MRLSM and the SSM at adjusted $\alpha$ reveal a close similarity in the vortices which emanate from the outboard leading edge, the inboard leading edge, the pylon, the nacelle, and the strake regarding their boundary layer interaction characteristics and their normalized vorticity $\omega / \omega_{\max , \text { Strake }}$ Furthermore, the extended flow separation for the SSM at unadjusted $\alpha$ shows a clear displacement of the strake vortex. The scaling effect is less pronounced compared to the difference between the LSM and the SSM at adjusted $\alpha$. Despite this, the results reaffirm that by modifying the SSM test toward smaller $\alpha$ by seeking $c_{\mathrm{L}}$-similarity of the LSM, an acceptable agreement in the vortical flow can be achieved. This finding is of high relevance when evoking the impact of the vortices on local flow separation.

\section{Conclusions}

A generic UHBR engine high-lift configuration is investigated in three different environments by means of RANS simulations. Those comprised the near full-scale open-jet wind tunnel T-101 at TsAGI, the small scale closed testsection wind tunnel at Tel-Aviv University, and an artificial environment introduced to simulate the small scale model (SSM) in a freestream surrounding. The purpose of this 
Fig. 15 Vortices in the slat cut-out region visualized using isosurfaces of $\lambda_{2}=-300$ colored with $\omega_{x}$
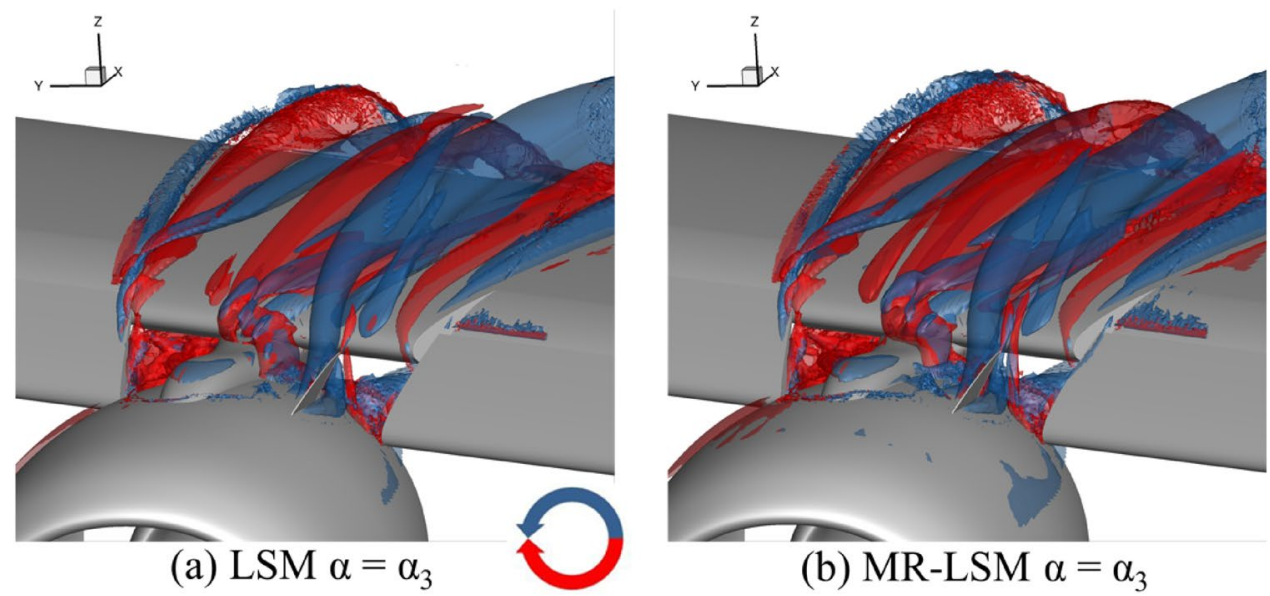

(b) MR-LSM $\alpha=\alpha_{3}$

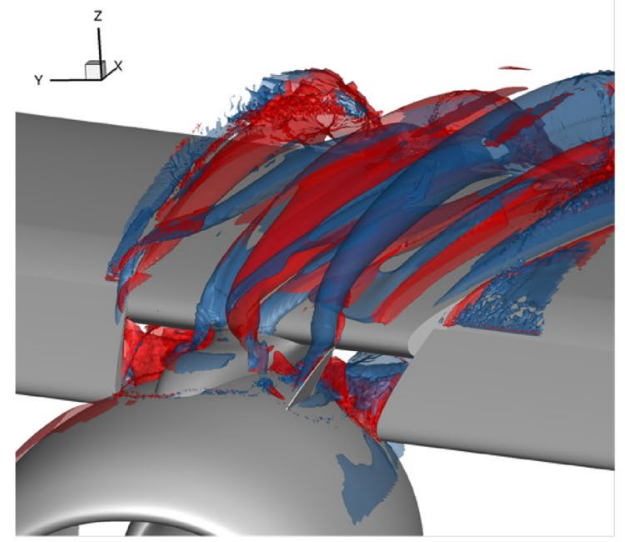

(c) $\operatorname{SSM} \alpha=\alpha_{1}-5^{\circ}$

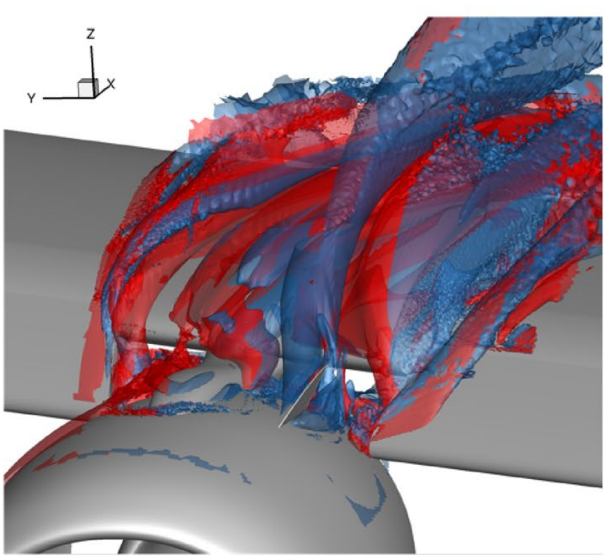

(d) $\operatorname{SSM} \alpha=\alpha_{3}$ study was to identify the individual impact of the Reynolds number and the wind tunnel walls on the attenuated flow downstream from the engine-nacelle and thus qualify small scale AFC tests on the configuration at Tel-Aviv University as a precursor for the large scale tests at TsAGI.

Extended validation with measurement data acquired from both wind tunnel tests show that the complex flow field in the slat cut-out region can be reproduced both qualitatively and quantitatively in an adequate manner by conventional CFD. This encouraged the cross-comparison of simulation results, which reveals a significant influence of the test section enclosure on the flow field around the small scale model. This could be attributed to the blockage effect and, for large incidence angles, to corner flow separation. On the other hand, the approximately eight-fold difference in Reynolds number shows an influence of far smaller order of magnitude. An implication of this is the possibility to derive a full-scale representative baseline flow on a small scale model for the investigated high-lift model without the need for cryogenic conditions.

Despite the significant wind tunnel wall effects on the small scale model, an approach was derived which allows to realize similar flow conditions in the slat cut-out region in terms of surface flow patterns, pressure distribution, and vortical flow. The latter finding is crucial regarding the transferability of the following AFC tests on the SSM within the ongoing project.

However, caution must be applied when considering general validity of the results due to the geometric peculiarities of the studied configuration. Furthermore, final confirmation of the findings requires test results of AFC applied on both the SSM and the LSM, as AFC interaction with external boundary layer is highly sensitive toward discrepancies in the local flow field, which were not analyzed in depth within this work. 
Fig. 16 Vortices in slat cut-out region visualized using contour planes of normalized vorticity parallel to the wing leading and volume streamlines

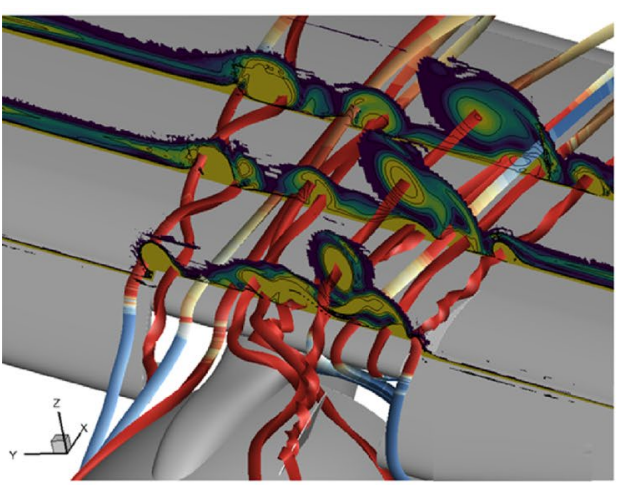

(a) $\operatorname{LSM} \alpha=\alpha_{3}$

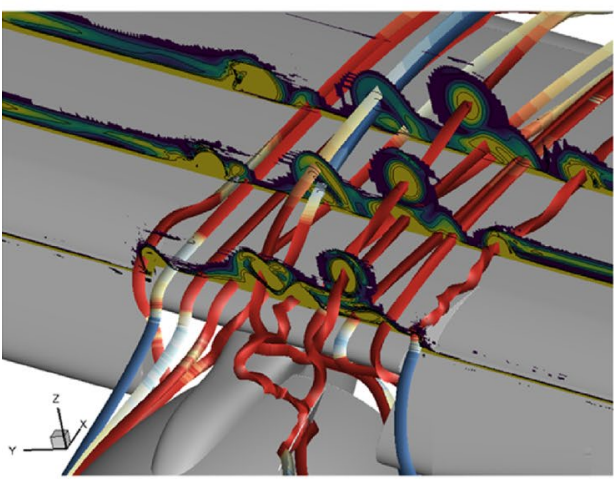

(c) $\operatorname{SSM} \alpha=\alpha_{1}-5^{\circ}$

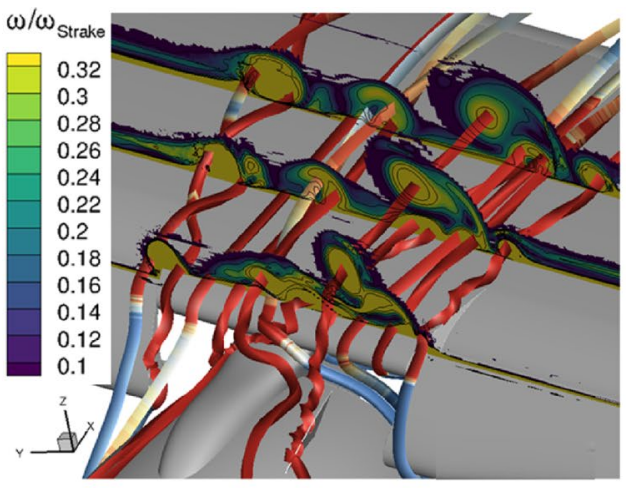

(b) MR-LSM $\alpha=\alpha_{3}$

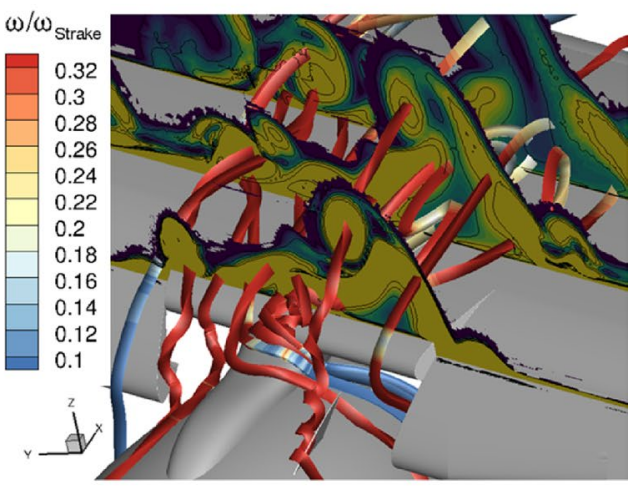

(d) $\operatorname{SSM} \alpha=\alpha_{3}$
Acknowledgements The work described in this paper has received funding from the European Commission program H2020-EU.3.4.5.1.IADP Large Passenger Aircraft, Grant agreement no. 754307, INAFLOWT project coordinated by Avraham Seifert and monitored by Bruno Stefes. Baseline data of the full-scale model were obtained during the measurement campaign of the AFloNext project within the European Commission Seventh Framework Program FP7/2007-2013, under Grant agreement no. 604013. The authors would like to express appreciation to all the participants of the INAFLOWT project for the great cooperation.

Funding This work was funded by Cleansky2. Open Access funding provided by Projekt DEAL.

Open Access This article is licensed under a Creative Commons Attribution 4.0 International License, which permits use, sharing, adaptation, distribution and reproduction in any medium or format, as long as you give appropriate credit to the original author(s) and the source, provide a link to the Creative Commons licence, and indicate if changes were made. The images or other third party material in this article are included in the article's Creative Commons licence, unless indicated otherwise in a credit line to the material. If material is not included in the article's Creative Commons licence and your intended use is not permitted by statutory regulation or exceeds the permitted use, you will need to obtain permission directly from the copyright holder. To view a copy of this licence, visit http://creativecommons.org/licenses/by/4.0/.

\section{References}

1. $\mathrm{CO}_{2}$ emissions from commercial aviation, 2018. The International Council on Clean Transportation (2018). https://theicct.org/sites /default/files/publications/ICCT_CO2-commercl-aviation2018_20190918.pdf. Online Accessed 01 July 2020

2. Kallas, S., Geoghegan-Quinn, M., Darecki, M., Edelstenne, C., Enders, T., Fernandez, E., Hartman, P.: Flightpath 2050 Europe's vision for aviation. Report of the High Level Group on Aviation Research, European Commission, Brussels, Belgium, Report No. EUR 98 (2011)

3. Allen, J.B., Oliver, W.R., Spacht, L.A.: Wind tunnel tests of highlift systems for advanced transports using high-aspect-ratio supercritical wings. NASA CR-3523 (1982)

4. Meyer, M., Bieler, H., Fricke, S., Wild, J., Norman, D.: Designing and testing active flow control systems at the junction of ultrahigh bypass ratio engines and the wing. ECCOMAS presentation (2014)

5. Cordis, INAFLOWT (2019). https://cordis.europa.eu/project/ rcn/210820_en.html. Online Accessed 21 Jan 2019

6. Arwatz, G., Fono, I., Seifert, A.: Suction and oscillatory blowing actuator. In: IUTAM Symposium on Flow Control and MEMS, pp. 33-44. Springer, Dordrecht (2008)

7. Cordis, AFLoNext (2019). https://cordis.europa.eu/project/ rcn/109010/reporting/en. Online Accessed 09 Feb 2019

8. Kintscher, M., Wiedemann, M., Peter, M.H., Heintze, O., Kühn, T.: Design of a smart leading edge device for low speed wind 
tunnel tests in the European project SADE. Int. J. Struct. Integr. 2(383-405), 11 (2011)

9. Wild, J.: Mach and Reynolds number dependencies of the stall behavior of high-lift wing sections. J. Aircr. 50(4), 1202-1216 (2013)

10. Hue, D., François, C., Dandois, J., Gebhardt, A.: Simulations of an aircraft with constant and pulsed blowing flow control at the engine/wing junction. Aerosp. Sci. Technol. 69, 07 (2017)

11. Soudakov, V., Amiryants, G., Schlösser, P., Bauer, M., Weigel, P., Bardet, M., Ciobaca, V., Gebhardt, A., Wild, J.: Full-scale windtunnel test of active flow control at the wing/pylon/engine junction. Presentation held at CEAS 2017, Bucharest, Romania

12. Schlösser, P., Soudakov, V., Bauer, M., Wild, J.: Active separation control at the pylon-wing junction of a real-scale model. AIAA J. 57(4), 132-141 (2019)

13. Fricke, S., Ciobaca, V., Wild, J., Norman, D.: Numerical studies of active flow control applied at the engine-wing junction. In: Advances in Simulation of Wing and Nacelle Stall. FOR 1066 2014. Notes on Numerical Fluid Mechanics and Multidisciplinary Design, vol. 131, pp. 397-411 (2016)

14. Barlow, J., Rae, H.W., Pope, A.: Low-speed wind tunnel testing. Wiley (1999)

15. Schwamborn, D., Gerhold, T., Heinrich, R.: The DLR TAU-code: recent applications in research and industry. In: ECCOMAS CFD 2006 Conference (2006)

16. Spalart, P.R., Allmaras, S.R.: A one-equation turbulence model for aerodynamic flows. In: 30th Aerospace Sciences Meeting and Exhibit, p. 439 (1992)

17. Togiti, V., Eisfeld, B., Brodersen, O.: Turbulence model study for the flow around the NASA common research model. J. Aircr. 51(4), 1331-1343 (2014)

18. Cary, A.W., Yousuf, M., Li, P., Mani, M.: Current practice unstructured grid CFD results for 3rd AIAA high lift prediction workshop. In: 2018 AIAA Aerospace Sciences Meeting, p. 1037 (2018)

19. Pointwise, Homepage (2019). https://www.pointwise.com/point wise. Online Accessed 15 Feb 2019
20. Vassberg, J.C., Tinoco, E.N., Mani, M., Rider, B., Zickuhr, T., Levy, D.W., Brodersen, O.P., Eisfeld, B., Crippa, S., Wahls, R.A., et al.: Summary of the fourth AIAA computational fluid dynamics drag prediction workshop. J. Aircr. 51(4), 1070-1089 (2014)

21. Vrchota, P., Prachař, A.: Active flow separation control applied at wing-pylon junction of a wing section in landing configuration. In: 55th AIAA Aerospace Sciences Meeting, At Grapevine, Texas, vol. 55 (2017)

22. Landa, T., Radespiel, R., Wild, J.: Simulation of longitudinal vortices on a high-lift wing. In: Advances in Simulation of Wing and Nacelle Stall - Notes on Numerical Fluid Mechanics and Multidisciplinary Design, vol. 131, pp. 351-366 (2016)

23. Fischer, O.: Correction Method for Interference Effects in OpenJet Wind Tunnels, pp. 25-40. Springer Fachmedien Wiesbaden, Wiesbaden (2018)

24. Eliasson, P.: Edge, a Navier-Stokes solver for unstructured grid. In: Proceedings to Finite Volume for Complex Applications III, pp. 527-534. ISTE Ltd., London (2002)

25. Ewald, B.F.R.: Wind tunnel wall corrections (la correction des effets de paroi en soufflerie). Technical report, Advisory Group for Aerospace Research and Development Neuilly-sur-Seine (France). No. AGARD-AG-336 (1998)

26. Rudnik, R., Germain, E.: Re-No. scaling effects on the EUROLIFT high lift configurations. In: 45th AIAA Aerospace Sciences Meeting and Exhibit, p. 752 (2007)

27. Lengers, M.: Industrial assessment of overall aircraft driven local active flow control. In: 29th Congress of the International Council of the Aeronautical Sciences, ICAS 2014 (2014)

28. Fisher, D., Cobleigh, B., Banks, D., Hall, R., Wahls, R.: Reynolds number effects at high angles of attack. In: 20th AIAA Advanced Measurement and Ground Testing Technology Conference, p. 2879 (1998)

Publisher's Note Springer Nature remains neutral with regard to jurisdictional claims in published maps and institutional affiliations. 